\title{
The CONSERT operations planning process for the Rosetta mission
}

\author{
Y. Rogez ${ }^{1}$, P. Puget ${ }^{2}$, S. Zine ${ }^{3}$, A. Hérique ${ }^{4}$, W. Kofman ${ }^{5}$ \\ Univ. Grenoble Alpes, CNRS, CNES, IPAG, F-38000 Grenoble, France \\ N. Altobelli ${ }^{6}$, M. Ashman ${ }^{7}$, M. Barthelemy ${ }^{8}$, M. Costa Sitjà ${ }^{9}$, B. Geiger ${ }^{10}$, B. Grieger ${ }^{11}$, \\ R. Hoofs ${ }^{12}$, M. Küppers ${ }^{13}$, L. O'Rourke ${ }^{14}$, C. Vallat ${ }^{15}$ \\ European Space Astronomy Centre/European Space Agency, PO. Box 78, 28691 Villanueva de la Cañada, Spain
J. Biele ${ }^{16}$, C. Fantinati ${ }^{17}$, K. Geurts ${ }^{18}$, M. Maibaum ${ }^{19}$, B. Pätz ${ }^{20}$, S. Ulamec ${ }^{21}$
Deutsches Zentrum für Luft und Raumfahrt. DLR-RB/MUSC, 51147 Köln, Germany \\ A. Blazquez ${ }^{22}$, C. Delmas ${ }^{23}$, J.-F. Fronton ${ }^{24}$, E. Jurado ${ }^{25}$, A. Moussi ${ }^{26}$ \\ Centre National d'Etudes Spatiales (CNES), 18 av. E. Belin, 31401 Toulouse, France \\ C.M. $\operatorname{Casas}^{27}$, A. Hubaultt ${ }^{28}$, P. Muñoz ${ }^{29}$ \\ European Space Operation Centre/European Space Agency, Germany \\ ESOC/ESA, Germany \\ and
}

${ }^{1}$ CONSERT Operation Engineer, Radar group

${ }^{2}$ CONSERT Project Manager, Radar group

${ }^{3}$ CONSERT Scientist, Planeto team, Radar group

${ }^{4}$ CONSERT Principal Investigator, Planeto team, Radar group

${ }^{5}$ CONSERT Principal Investigator until 2018, Planeto team, Radar group

${ }^{6}$ Rosetta Scientist, RSGS

${ }^{7}$ Rosetta Science Operations Engineer, RSGS

${ }^{8}$ Rosetta Archive and Liaison Scientist, RSGS

${ }^{9}$ SPICE and Auxiliary Data Support Engineer, RSGS

${ }^{10}$ Rosetta Liaison Scientist, RSGS

${ }^{11}$ Rosetta Trajectory Design Scientist, RSGS

${ }^{12}$ Rosetta Science Operations Advisor, RSGS

${ }^{13}$ Rosetta Scientist, RSGS

${ }^{14}$ Rosetta Downlink Science Operations Manager, RSGS

${ }^{15}$ Rosetta Scientist, RSGS

${ }^{16}$ Philae Scientist and Payload Manager, RB-MUSC

${ }^{17}$ Philae Operations Manager, LCC

${ }^{18}$ Philae Technical Manager, LCC

${ }^{19}$ Philae Systems Engineer, LCC

${ }^{20}$ Philae LCC Manager, LCC

${ }^{21}$ Philae Project Manager, LCC

${ }^{22}$ Philae Flight Dynamics Engineer, SONC

${ }^{23}$ Philae Science Operations Engineer, SONC

${ }^{24}$ Philae Science Ground Segment, SONC

${ }^{25}$ Philae Flight Dynamics Engineer, SONC

${ }^{26}$ Philae Science Operations Engineer, SONC

${ }^{27}$ Rosetta Flight Dynamics Engineer, RMOC

${ }^{28}$ Rosetta Operations Engineer, RMOC

${ }^{29}$ Rosetta Flight Dynamics Engineer, RMOC

American Institute of Aeronautics and Astronautics 
R. Hahnel ${ }^{30}$, D. Plettemeier ${ }^{31}$

Technische Universitaet Dresden Helmholtzstraße 10 D-01069 Dresden, Germany

In the scope of European Space Agency's Rosetta mission, the COmet Nucleus Sounding Experiment by Radio wave Transmission (CONSERT) has sounded the deep interior of the nucleus of comet 67P/Churyumov-Gerasimenko. The CONSERT experiment main objective was to image the interior of the comet nucleus.

This bi-static radar experiment with instrument units on-board both, the Rosetta main spacecraft and its lander Philae, requires a specific geometric configuration to operate and produce fruitful science data. Thus, these geometric constraints involve mainly the position and orientation of Rosetta and Philae. From the operations planning point of view, the mission constraints imposed observation slots to be defined far before their execution, while the comet shape, spacecraft trajectories and landing site were still unknown. The CONSERT instrument operations scheduling had to be designed jointly for Rosetta and Philae platforms, based on different time scales and planning concepts.

We present the methods and tools we developed to cope with the complexity of this planning process. These operations planning concepts allowed handling the complexity of multiple platform operations and the lack of prior knowledge of the observed target.

\begin{tabular}{ll} 
& \multicolumn{1}{c}{ Nomenclature } \\
$67 P / C-G$ & $=$ Comet 67P/Churyumov-Gerasimenko \\
$C P U$ & $=$ Central Processing Unit \\
$f$ & $=$ Clock frequency \\
$E S A$ & $=$ European Space Agency \\
$F S S$ & $=$ First Science Sequence (Philae's mission phase) \\
$H P C$ & $=$ High Performance Computer \\
$L C C$ & $=$ Lander Control Center, DLR (Köln) \\
$L C N$ & $=$ CONSERT Lander unit \\
$L T S$ & $=$ Long Term Science (Philae's mission phase) \\
$L D P$ & $=$ Landing Delivery Preparation (Philae's mission phase) \\
$L S S P$ & $=$ Landing Site Selection Process (Philae's mission phase) \\
$N A I F$ & $=$ NASA's Navigation and Ancillary Information Facility \\
$N A S A$ & $=$ National Aeronautics and Space Administration \\
$O C N$ & $=$ CONSERT Orbiter unit \\
$R L G S$ & $=$ Rosetta Lander Ground Segment, composed of SONC and LCC \\
$R M O C$ & $=$ Rosetta Mission Operation Center, ESOC (Darmstadt) \\
$R S G S$ & $=$ Rosetta Science Ground Segment, ESAC (Madrid) \\
$S D L$ & $=$ Separation Descent and Landing (Philae's mission phase) \\
$S O N C$ & $=$ Philae Science Operation and Navigation Centre, CNES (Toulouse) \\
$S P I C E$ & $=$ NAIF observation geometry system for space science missions \\
$W o O$ & $=$ CONSERT Window of Opportunity \\
$Z_{L D R}$ & $=$ Philae local $+Z$ axis, pointing towards its top panel \\
$Z_{O R B}$ & $=$ Roestta spacecraft local +Z axis, pointing towards the target
\end{tabular}

${ }^{30}$ CONSERT Antenna System Engineer, Institut für Nachrichtentechnik

${ }^{31}$ CONSERT Co-Inverstigator, Institut für Nachrichtentechnik

American Institute of Aeronautics and Astronautics 


\section{Introduction}

From July 2014 until September 2016, the European Space Agency's mission Rosetta observed in-situ the nucleus and the environment of comet 67P/Churyumov-Gerasimenko (67P/C-G). It is a Jupiter-family comet with a diameter of about $3.8 \mathrm{~km}$ and a rotation period of about 12.4 hours.

This mission led to the most detailed study of a comet ever. The Rosetta orbiter monitored the comet activity, measured the composition of its environment and characterized the nucleus surface structure and composition. The Rosetta lander, Philae, landed on the nucleus on the $12^{\text {th }}$ of November 2014, acquired images and obtained information on the composition and the physical properties of the surface material at the landing site location ${ }^{1,2}$. These close observations of this primitive building block of the Solar System help to understand its formation and evolution.

The Rosetta spacecraft was launched on the $2^{\text {nd }}$ of March 2004 with a scientific payload composed of eleven instruments including Philae, also embedding ten other instruments. After several gravity assist manoeuvres around the Earth and Mars, the fly-by observations of asteroids 2867/Šteins and 21/Lutetia, Rosetta entered a two-year deep space hibernation period in June 2011 then woke up in January 2014 for its rendezvous with the comet. Scientific investigations, orbiting around the nucleus and coma, were performed continuously until the $30^{\text {th }}$ of September 2016, when the spacecraft accomplished a controlled impact on the surface ${ }^{3}$.

The COmet Nucleus Sounding Experiment by Radiowave Transmission (CONSERT) was one of these payload instruments. It was a bi-static low frequency radar composed of two units: one on-board the Rosetta orbiter and the other on-board Philae (Figure 1). It was the only instrument which performed a systematic synchronous operation of both orbiter and lander platforms.

The science objective of the CONSERT instrument was to provide information about the nucleus deep interior. It allowed to deduce the physical characteristics of the comet nucleus ${ }^{4}$ and to constrain its internal composition ${ }^{5}$. CONSERT could have discriminated between different hypotheses on comet nucleus structural models by imaging its interior, although this has not been possible due to the unexpected landing conditions of Philae.

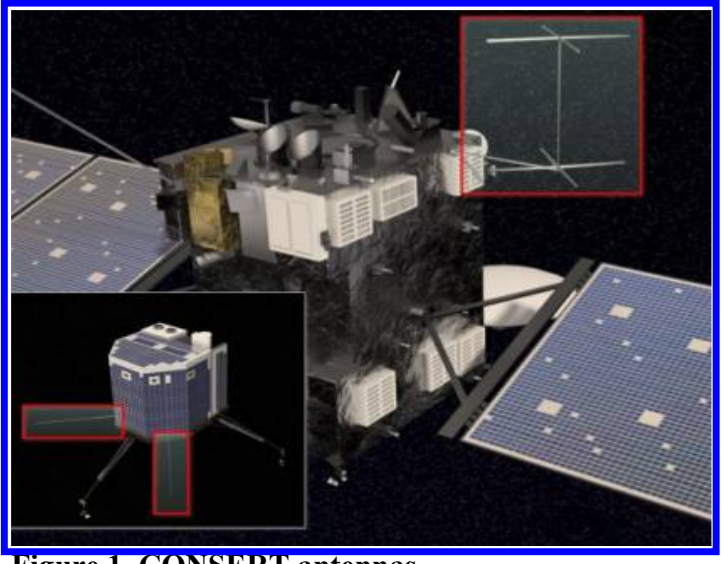

Figure 1. CONSERT antennas

The CONSERT instrument has also been used to support Rosetta orbiter and lander operations. Indeed, the bi-static radar ranging allowed a first localization of the final lander's touchdown position ${ }^{6}$. It also performed measurements between the orbiter and the lander to help the reconstruction of the trajectory and attitude of Philae ${ }^{7}$ during the descent to the comet surface.

This paper describes the very specific methods and tools developed by the CONSERT team in collaboration with all platform teams needed to manage a particularly complex operations scheme. It presents the instrument specific operational constraints, the Rosetta planning process logic and the selected operational solution and implemented tools. Finally, the paper depicts the CONSERT team's perspectives and current work based on this experience.

American Institute of Aeronautics and Astronautics 


\section{CONSERT functioning and operation}

The CONSERT instrument measures a $90 \mathrm{MHz}$ wave propagation delay, the signal amplitude and polarization in transmission through the comet nucleus. For each acquisition point, also called a "sounding", the phase-modulated coded signal is transmitted by the CONSERT lander unit (LCN) from Philae, landed on the surface and received by the CONSERT orbiter unit $(\mathrm{OCN})$ on Rosetta spacecraft, orbiting around the comet body (Figure 2).

The travel time of the radio wave through the comet nucleus medium is delayed as compared to the travel time in vacuum. This delay is directly related to the mean dielectric permittivity of the internal medium along the propagation path. The absorption and signal shape provide additional information on the material physical properties. This measurement is performed regularly along the Rosetta orbiter trajectory. The repetition of these acquisitions for several and various trajectories of Rosetta spacecraft allows a threedimensional radar imaging of the interior of the nucleus (Figure 3).

The CONSERT propagation delay accuracy is directly linked to the synchronized functioning of the instrument internal clocks on OCN and LCN. The time measurement shall be better than $0.1 \mu \mathrm{s}$, which leads to a high constraint on the clock's frequency stability over a whole acquisition sequence $\left(\Delta \mathrm{f} / \mathrm{f}=10^{-12}\right.$ over typically 10 hours) and a precise synchronization between them. To achieve this technically unreachable constraint in a relatively small instrument, the system made use of the time-transponder technique. At each sounding, OCN transmits the signal to LCN for synchronization: the "ping". Then LCN mirrors it to OCN: the "pong". The "pong" follows exactly the same path as the "ping" because we can consider that the relative position of Rosetta is fixed at this timescale. In that way, the OCN unit is able to measure twice the propagation delay plus the mirror processing system delay on LCN (Figure 4).

Using this method, the instrument still needs a tuning phase prior to the acquisition but with a frequency stability constraint of only $\Delta \mathrm{f} / \mathrm{f}=10^{-7}$ over the sequence ${ }^{8}$.

The CONSERT signal amplitude and polarization is mainly driven by the properties of its antennae. LCN antenna consists of two orthogonal monopoles in front of Philae's body. It can generate a left-handed elliptic polarized wave. OCN is made of two pairs of two cross dipoles, also providing a left-handed elliptic polarized wave ${ }^{9}$. When nominally landed, which was, unfortunately, definitely not the case for the 2014 landing ${ }^{10}$, the main part of the LCN transmitted signal power is directed to the ground, due to coupling with the ground and the platform structure ${ }^{11}$. This ensures the best link budget during the science operations when

American Institute of Aeronautics and Astronautics

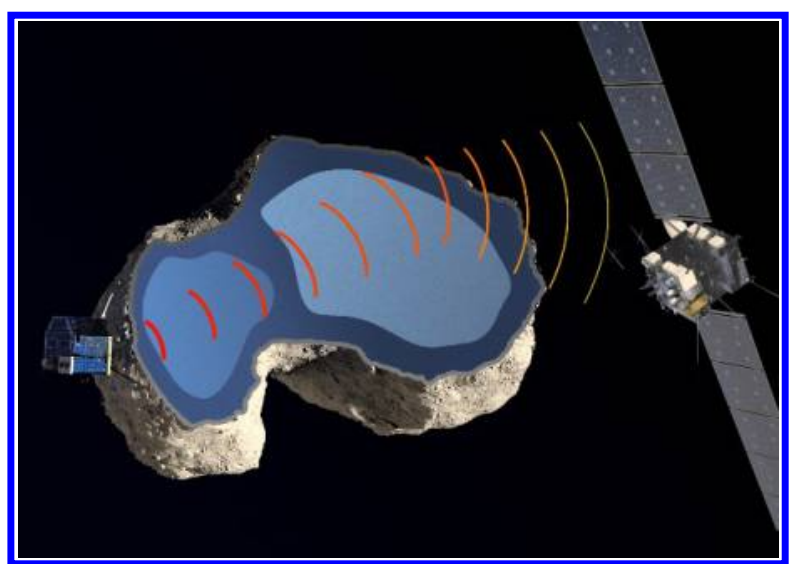

Figure 2. CONSERT instrument concept. The radio wave propagates through the comet nucleus between the lander and the orbiter units.

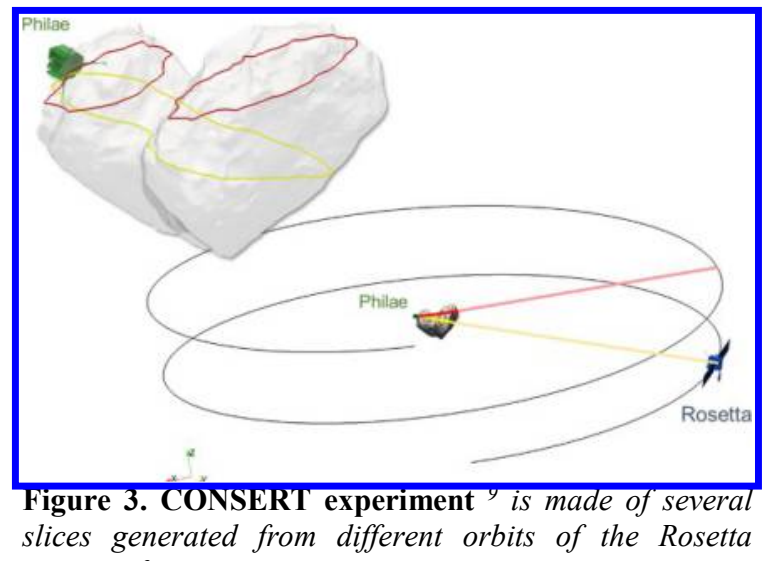
spacecraft.

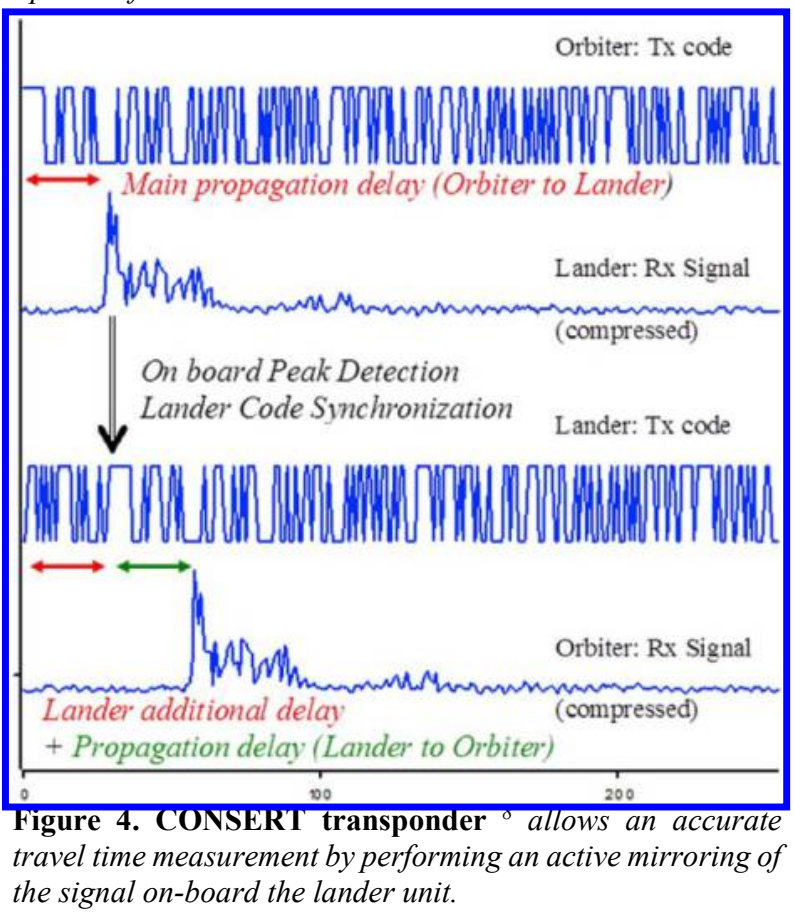


penetrating through the comet nucleus, but makes this link more difficult when OCN and LCN are operated in visibility.

The CONSERT complete science acquisition sequence is composed of five phases ${ }^{12}$, taking place along the Rosetta spacecraft trajectory. They are described in Table 1.

Table 1. CONSERT operation sequence phases.

\begin{tabular}{|c|c|c|c|}
\hline $\begin{array}{c}\text { CONSERT } \\
\text { operation phase }\end{array}$ & Purpose and Description & Configuration & $\begin{array}{l}\text { Typical } \\
\text { duration }\end{array}$ \\
\hline Warm-up & $\begin{array}{l}\text { To put the instrument in the right } \\
\text { functioning temperature and clock } \\
\text { stabilization. }\end{array}$ & Any. & $1 \mathrm{~min}$ \\
\hline Tuning & $\begin{array}{l}\text { To match the frequency of the clocks on } \\
\text { both CONSERT units. }\end{array}$ & $\begin{array}{l}\text { Direct visibility between OCN } \\
\text { and LCN. }\end{array}$ & $6 \mathrm{~min}$ \\
\hline Waiting & $\begin{array}{l}\text { To wait for the beginning of the nucleus } \\
\text { sounding. Signal transmission is interrupted } \\
\text { to optimize the overall power consumption. }\end{array}$ & $\begin{array}{l}\text { Direct visibility to comet } \\
\text { nucleus occultation. }\end{array}$ & $2 \mathrm{hrs}$ \\
\hline Sounding & $\begin{array}{l}\text { To perform the CONSERT science } \\
\text { measurements. }\end{array}$ & $\begin{array}{l}\text { OCN and LCN units are } \\
\text { occulted by the comet nucleus. }\end{array}$ & $8 \mathrm{hrs}$ \\
\hline Calibration & $\begin{array}{l}\text { Optionally, to perform a background noise } \\
\text { measurement in the same environmental } \\
\text { conditions as the soundings. }\end{array}$ & Direct visibility. & $20 \mathrm{~min}$ \\
\hline
\end{tabular}

In order to perform this science operation sequence in proper conditions for various trajectories of the Rosetta spacecraft, one had to make sure that a set of technical constraints were fulfilled ${ }^{9}$. Most of these radar constraints could be derived in the end into geometrical ones, involving Rosetta, Philae and 67P/C-G relative positions and orientations. To perform good science acquisitions, both CONSERT units clocks must be synchronized, which implies the tuning phase to be successfully completed. As Philae is fixed on the nucleus surface, this mainly induces geometrical constraints on the Rosetta orbiter platform. To maintain a sufficient signal to noise ratio, and thus a good link budget, the distance between Philae and Rosetta is also constrained, as well as their relative position and attitude, to ensure a good configuration of the two antenna lobes. The motion of the Rosetta spacecraft relative to the comet nucleus surface is dominated by the comet revolution. Thus, a measurement sequence naturally covers all the longitudes. It is mainly parameterized by the orbiter latitude. To ensure that the orbiter is static over a whole "ping-pong", the footprint velocity must be limited, which also implies a certain constraint on the spacecraft distance to lander.

In addition, the required geometry configuration between Rosetta, Philae and 67P/C-G is constrained by the necessity of OCN to receive the LCN signal throughout the nucleus. Because of its global shape, the comet nucleus focuses the quasi-omnidirectional wave transmitted by LCN. Therefore, OCN must be located such that it is inside the CONSERT signal wave front (Figure 5), which

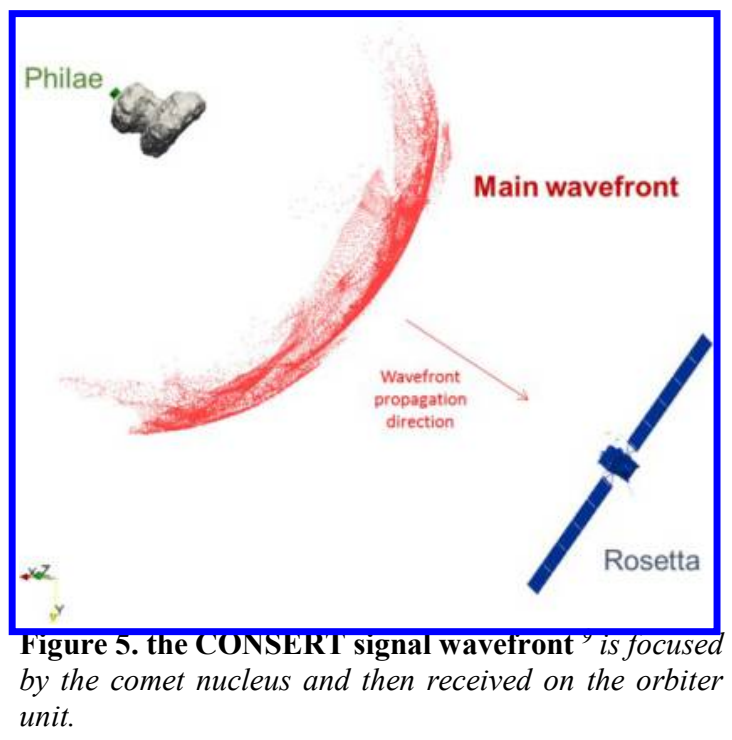
corresponds to a restrained portion of the sky at the opposite side of the comet with regard to Philae's landing location.

Furthermore, the CONSERT operations team must verify that its instrument can be fed with telecommands and sufficient power on lander side. Philae was powered mainly by its batteries, which could be recharged by its solar panels. Thus, we must check that the lander platform was illuminated by the Sun for a sufficient period prior to

American Institute of Aeronautics and Astronautics 
beginning any payload operation. In addition, the pre-requisite to receive operational commands and to allow time updates from the orbiter had to be considered.

Finally, the radar signal could be perturbed by other sub-systems and payloads of Rosetta or Philae. To maintain a good quality of the CONSERT signal, we had to ensure that these sub-systems were not operated at the same time as our instrument.

All these operational constraints had to be checked and fulfilled for a successful performance of the CONSERT experiment during the Rosetta mission. The resolution of this whole set of requirements for each science sequence was particularly challenging and required the definition of specific planning processes and tools.

\section{Operations planning process}

The CONSERT operations were limited by Philae's end of mission date, initially foreseen in March 2015. Therefore, the CONSERT operations planning nominally covered a period of 6 months. The Philae mission was split into five phases, inside which CONSERT team defined its observation strategy, described in Table 2. Apart from this foreseen strategy, the CONSERT instrument was also been operated following the unexpected landing and during the LTS. By performing ranging measurements, Philae's final touchdown location zone could be determined and finally imaged by the OSIRIS camera ${ }^{10}$.

Table 2. CONSERT science observations strategy

\begin{tabular}{|c|c|}
\hline $\begin{array}{c}\text { Lander mission } \\
\text { phase }\end{array}$ & CONSERT activities \\
\hline $\begin{array}{l}\text { LSSP } \\
\text { Landing Site } \\
\text { Selection Process }\end{array}$ & $\begin{array}{l}\text { CONSERT team was deeply involved in the analysis, the ranking and the final selection } \\
\text { of the Philae landing site. Indeed, the position of Philae on the nucleus surface, which } \\
\text { was fixed all over the mission, was of crucial importance for CONSERT operations. } \\
\text { Unfortunately, Philae did not land nominally and the actual science operations had to be } \\
\text { shortened leading to a more difficult analysis of the data, obtained from the CONSERT } \\
\text { instrument. In any case, at the time of the planning of operations, we had to consider the } \\
\text { optimal trade-off for the landing site. }\end{array}$ \\
\hline $\begin{array}{l}\text { LDP } \\
\text { Landing Delivery } \\
\text { Preparation }\end{array}$ & $\begin{array}{l}\text { We performed calibration operations with Philae still attached to Rosetta. We took the } \\
\text { opportunity to be close to the comet surface to attempt sounding with CONSERT } \\
\text { operating as a mono-static low frequency radar. }\end{array}$ \\
\hline $\begin{array}{l}\text { SDL } \\
\text { Separation, Descent } \\
\text { and Landing }\end{array}$ & $\begin{array}{l}\text { CONSERT was operated to monitor the relative position and attitude all along the } \\
\text { descent. This also was an opportunity to sound the surface when Philae was close enough } \\
\text { to the surface. }\end{array}$ \\
\hline $\begin{array}{l}\text { FSS } \\
\text { First Science } \\
\text { Sequence }\end{array}$ & $\begin{array}{l}\text { On the } 12^{\text {th }} \text { of November } 2014 \text {,shortly following the assumed (final) landing, CONSERT } \\
\text { was operated successfully for } 10.5 \text { hours of science acquisition. This was the main } \\
\text { operations in the CONSERT observation strategy to ensure a first slice nucleus deep } \\
\text { sounding. }\end{array}$ \\
\hline $\begin{array}{l}\text { LTS } \\
\text { Long Term Science }\end{array}$ & $\begin{array}{l}\text { This phase was planned with an unknown end date, depending on many unpredictable } \\
\text { cometary environment conditions. Considering the unexpected landing, it finally did not } \\
\text { happened in practice. At the time of the mission planning process, this could not be } \\
\text { considered. So, for CONSERT operational planning, this phase was dedicated to sound } \\
\text { more slices of the nucleus, by performing as many of the science sequences as possible. } \\
\text { This paper describes specifically the operations scheduling activities for this period. }\end{array}$ \\
\hline
\end{tabular}

This observation strategy had to be implemented before any landing site selection was possible and without knowledge of the actual comet shape. The situation was even more demanding; due to the complexity of the Rosetta mission itself, involving a lot of instruments, observation requirements and the challenges of unprecedented navigation manoeuvres around a cometary nucleus. The planning of CONSERT operational activities had to be performed in collaboration with the four Rosetta operations centres, since CONSERT was both a lander and an orbiter payload. Each centre (Table 3) had specific requirements and different planning logic and priorities. 
The orbiter planning process, defined by the RSGS, consisted of a series of planning periods, each of which progressively refined the definition of operations. The global planning loop started approximately 6 months prior to the operations, with a quasi-final definition of the telecommands two weeks before their execution ${ }^{13}$. This planning process involved many actors: all orbiter platform and payloads teams. It allowed a smooth definition of the dense and complex sequence of operations for Rosetta. These planning activities started very early and lasted a long time before the execution of actual operations on-board. They had to be initiated without any knowledge of the 67P/C-G shape model and Philae landing site.

On the Philae side, operations planning was naturally designed around the critical landing event. As for CONSERT on the orbiter side, a RSGS liaison scientist relayed the operation requests to the orbiter planning, in order to coordinate the Philae and Rosetta operations ${ }^{14}$. Most of the lander payload operations did not depend on Rosetta trajectories, apart from CONSERT. Thus, the Philae planning process was conducted over a significantly shorter timeframe. For this reason, it was particularly difficult for the CONSERT team to cope with both planning concepts.

The cornerstone of the CONSERT operations planning was the landing of Philae. For operations directly following this event, we had to furnish to RSGS our observation requests even before the completion of the landing site selection 15, 16. To do so, we decided to proceed by overbooking the CONSERT operations schedule, including a lot of margin, then de-scoping and progressively refining these margins. This needed a significant amount of extra-work in trajectory analysis because we had to take into account all candidate landing sites and environmental conditions for Philae. Then we defined CONSERT windows of opportunities (WoO), and ranked them with scientific and technical criteria. At the very last moment, when the Philae landing site has been defined and Rosetta trajectory fixed, we de-scoped from the orbiter schedule all CONSERT WoOs that became irrelevant.

With this method, we could have prepared all the LTS period, taking into account all CONSERT instrument constraints and furthermore, iteratively negotiated and scheduled in collaboration with all other platform and instruments teams. 


\section{Operational tools}

Considering all the CONSERT operational constraints and the organizational complexity of the Rosetta mission, we strongly needed a set of automated tools to support the planning activities (Figure 6).

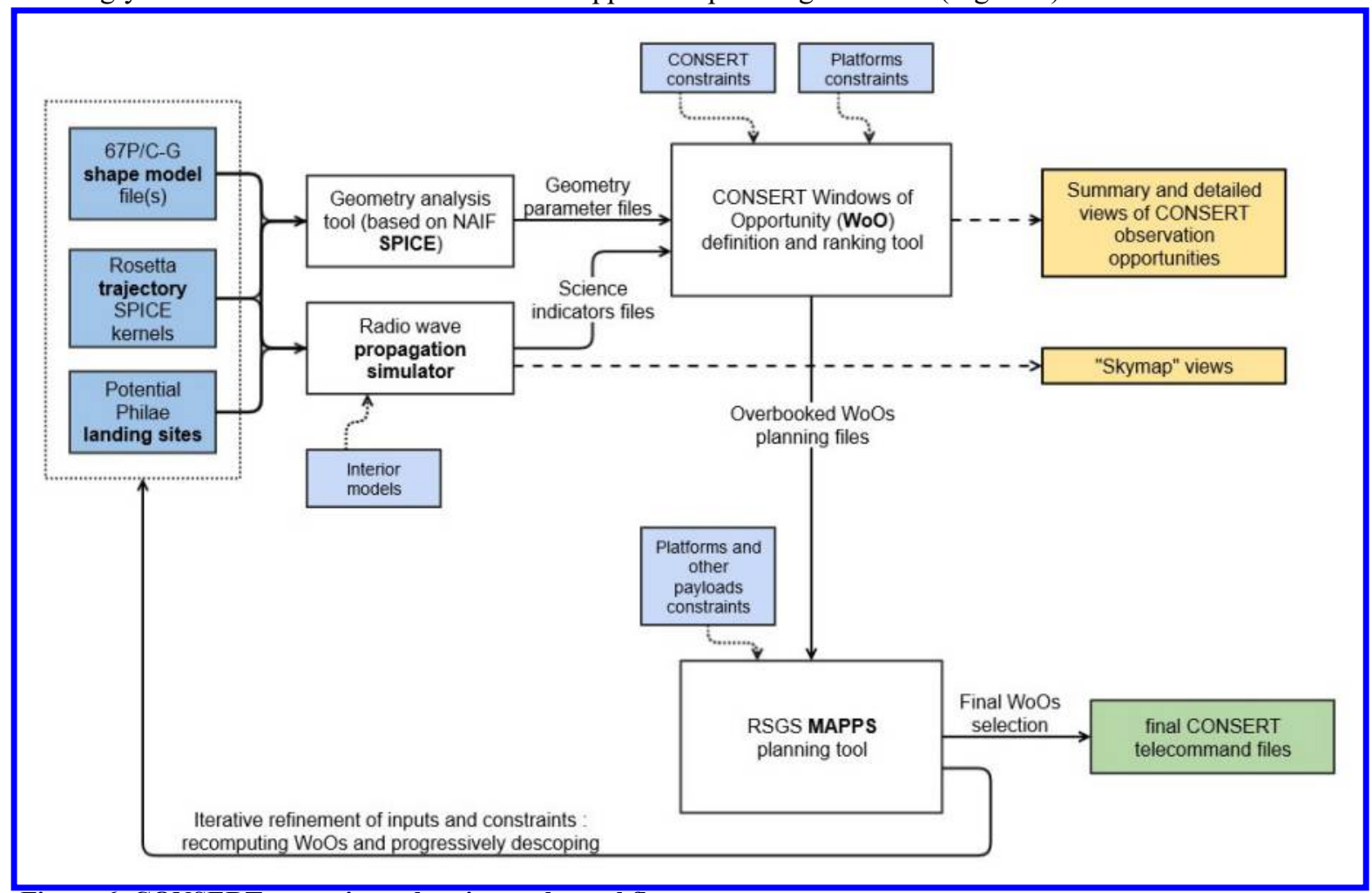

Figure 6. CONSERT operations planning tools workflow

The main inputs of this CONSERT planning tools workflow were in constant evolution and refinement through the Rosetta and Philae planning period. They consisted of the comet shape models provided by OSIRIS and RMOC, the Rosetta trajectory Spice kernels provided by RSGS and RMOC and the Philae potential landing sites provided by SONC. The combination of all these inputs defined a single CONSERT observation context, for which a set of opportunities could be identified. One can imagine that the total number of covered cases and computations performed by the geometry analysis and wave propagation tools was very large and required a significant amount of CPU time. Obviously, this had to be done by a fairly automated workflow.

The CONSERT operations are strongly driven by the geometrical configuration of the observation. A specific tool was in charge of the systematic computation of the geometrical parameters of interest. It was based on the NAIF Spice library, in accordance with RSGS processes. Taking as input the Spice kernels of the Rosetta trajectory, the Spice shape model kernels and the Philae landing site position, it computed and output in dedicated files up to 24 geometric values as a function of the UTC time. The most important variables were the distance between the lander and the orbiter, the angle between the lander and orbiter boresight directions (the emission angle), the visibility status between the two units, and the illumination of the lander by the Sun. RSGS supported CONSERT by producing a partial set of the orbiter-based parameters, which allowed a cross-validation of results. Similarly, SONC computed "event files" which included UTC timestamps for critical operational events (for example the switch from Sun illuminated to notilluminated state for the lander). This also allowed an additional validation of the results.

These parameters were directly linked to the resolution of the CONSERT operational constraints. To get a first rough approximation of the CONSERT science efficiency, we also computed the distance travelled inside the nucleus by a straight line between Rosetta and Philae. To refine this information, we flagged this "section" indicator to specify which region of the nucleus is sounded: the small lobe of the nucleus, the big lobe or both.

In parallel, the same input data were processed by a CONSERT experiment simulator, called SimSERT. This computationally intensive software, run on a HPC system, was able to simulate the physics of the CONSERT experiment and to give a sufficiently accurate estimation of the signal output. This numerical simulation is based on

American Institute of Aeronautics and Astronautics 
a ray-tracing algorithm, assuming a homogeneous interior model, with a given relative permittivity. Its output was post-processed to derive the time during which Rosetta crossed the simulated CONSERT signal along its trajectory. This is directly linked to the efficiency of an observation in terms of science return, so it provided a good global indicator for the quality of an acquisition sequence. The SimSERT software also exported special quick and comprehensive views of a combination of the computation results, called "skymaps" (Figure 7), which included also the SONC event files information. These views were of significant importance to support the CONSERT team in the evaluation of a high number of possibilities, keeping all useful details for proper decision-making.

All the parameters computed by the Spice tool and SimSERT were passed to the "WoO" (Window of Opportunity) tool, as data files. The $\mathrm{WoO}$ generator had the responsibility of the identification and characterization of the temporal slots in which CONSERT observations were possible.

All the CONSERT constraints as discussed above were implemented in this program, using customizable values. The geometrical parameters were then used to determine whether the technical constraints could be fulfilled or not. As an example, the Sun illumination angle was derived into beginning and ending UTC times between which the lander batteries are operational, the emission angle was derived into beginning and ending UTC times between which the CONSERT tuning is possible, etc... So, for each CONSERT possible operations opportunity, we could have generated detailed chronograms including comprehensive science indicators like the nucleus section crossed by the signal, the duration of the simulated useful signal duration, the latitude of the spacecraft, etc...

All the observation sequences opportunities, for all the possible combinations of Philae location and Rosetta trajectories, were then evaluated automatically to eliminate the unambiguously impossible ones. The ones that seem promising to the system were green-lighted. The remaining intermediate WoOs were also flagged, for further analysis by the researchers. For those cases, the tool also offered detailed plots and full data table views of the parameters evolutions. This allowed a first selection of the WoOs. The CONSERT team researchers then put a rank number for each, according to the science return. This enhanced list of WoOs was then sent to the LCC team for a lander operational evaluation of the selected WoOs. The LCC engineers then also ranked, validated or invalidated the WoOs on an operational complexity point of view. This operational complexity evaluation was also helped by the system, being able to identify six operational configurations. These configurations classify the quality and difficulty for operating Philae for CONSERT.

The final output of the WoO tools were summarized views (Figure 8) of all the WoOs in a single chronogram. This allowed the scientists and engineers to get a global view on a long period of operation, in one sight.

Once the ranking of the WoOs has been made by CONSERT and LCC teams, the remaining ones were implemented into RSGS planning files. The consecutive selected WoOs were merged into single corresponding operation requests ("FOT2", "FOT3" indicated as yellow boxes in Figure 8). Then, they were ingested into the MAPPS ${ }^{17}$ tool to allow interactions with all other orbiter payloads. MAPPS also provided very useful verification routines on constraints, especially with regard to the instruments conflicts detection on pointing requests and interferences.

During the last weeks of the Rosetta planning process, the merged CONSERT operations requests were iteratively reduced to single WoOs (corresponding to one of the green box on Figure 8, for each yellow slot). The final telecommands were finally implemented and verified by RMOC, one week before their in-flight execution.

American Institute of Aeronautics and Astronautics 


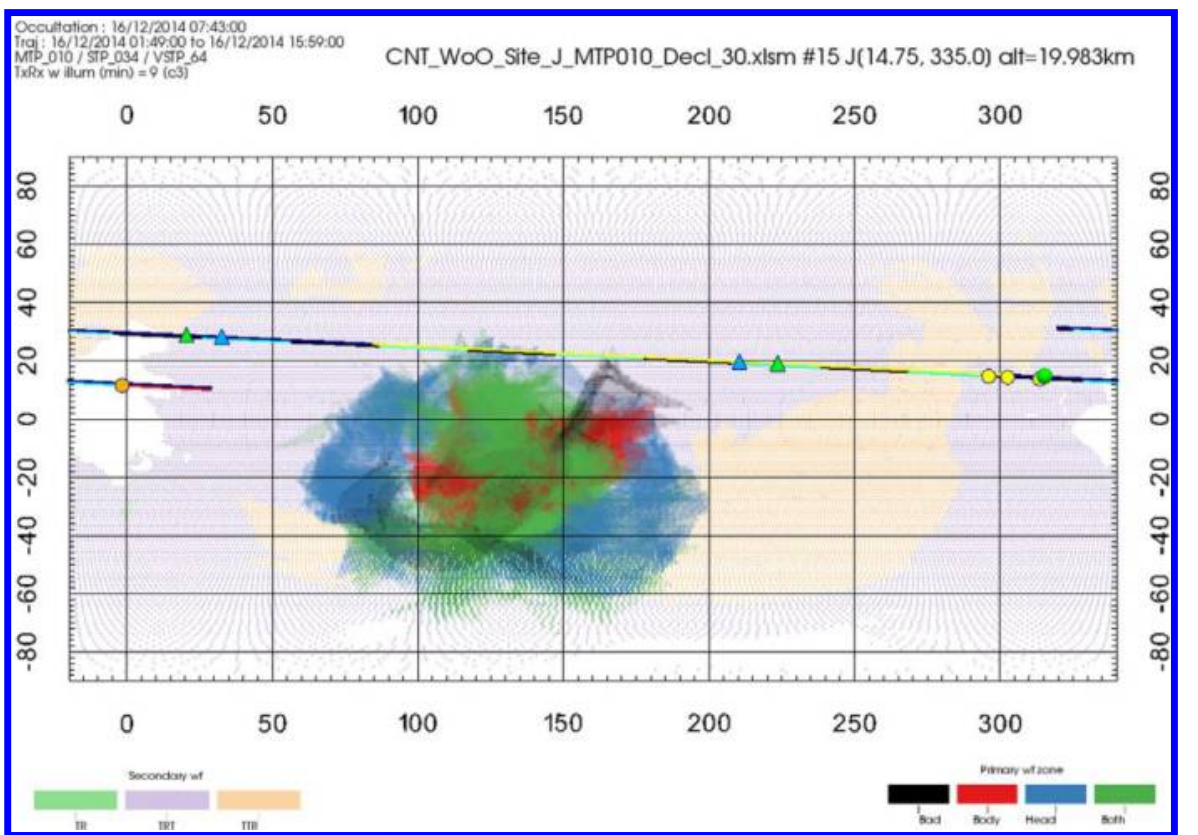

Figure 7 . a CONSERT "skymap" sample" in longitude and latitude in the comet nucleus fixed frame. The colored dots composing the background show the CONSERT signal, as transmitted by the lander unit. Their color map the internal region inside the nucleus that the signal traverses. The most saturated dots correspond to the most powerful signal. The colored line represents the track of Rosetta. Consequently, the more the line passes through the signal, the more efficient the CONSERT acquisition is. The colored triangles and circles along the Rosetta trajectory depict the constraints events, e.g. when Philae batteries could be loaded or when the CONSERT tuning phase is possible.

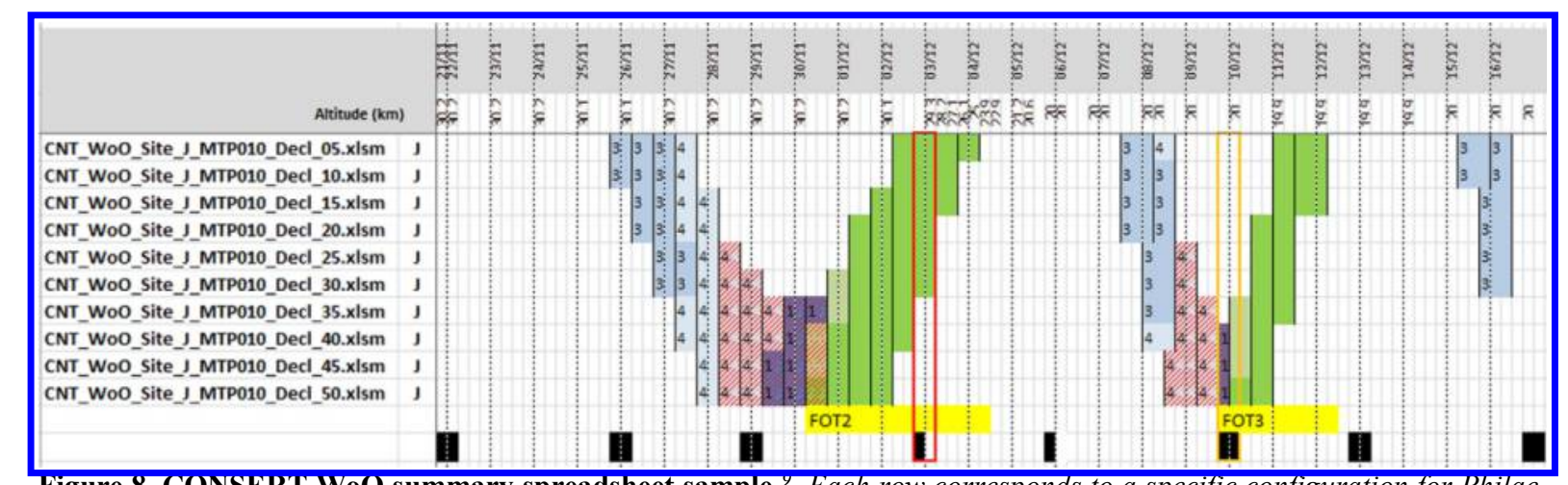

Figure 8. CONSERT WoO summary spreadsheet sample ${ }^{y}$. Each row corresponds to a specific configuration for Philae, the comet and Rosetta. The columns are the time calendar for one month of operations. Green boxes shows the time span where CONSERT operations are possible with a good science return. Other colors highlight different constraints inadequacy, showing the complexity value of the Philae operation. Black boxes, red and yellow outlines show periods during which operations are not possible because of Rosetta maneuvers. The yellow boxes then show, for a sweep of acquisition conditions, the window of opportunity for CONSERT observation, which will be refined progressively until the actual operations, finally corresponding to one or two specific green boxes.

American Institute of Aeronautics and Astronautics 


\section{Conclusion}

The Rosetta planning concept could only be finalized just approximately two years before the Philae landing. That meant a relatively short time for the implementation of our tools. Most of them were designed as ad-hoc prototypes. However, they met the requirements and proved their efficiency. They have also provided a valuable experience for the design and implementation of ground segment equipment for the next generation of radars for small bodies' observations in the Solar System.

It is recommended that the analysis of operational constraints and the first prototypes of operations planning tools, in terms of trajectory and science return, shall be assessed in the first payload development phases. This should be facilitated by the usage of programming and data standards as NAIF Spice kernels or shape model files. The very early predictions on operational scenarios can be far from reality, but the general planning process and tools most probably would remain valid. These tools might be able to handle their tasks with a great margin. For instance, in our case, the unexpectedly complex shape of the 67P/C-G's nucleus discounted some analyses that were based on the assumption of a quasi-ellipsoidal body.

The planning and operational processes should also be defined early in the project development, to allow the payload teams to design and implement robust and automated systems. With the Rosetta mission, and more specifically with the CONSERT lander-orbiter combined instrument, new tools and processes have been used for human and machine interactions. In this regard, timeliness is of major importance when defining a project's wide procedures and file format standards. In this regard, ESA is offering today opportunities to payload collaborators for training in use of the Spice Toolkit libraries. It also continues the development of the MAPPS tool ${ }^{17}$, generalizing its usage on current and future planetary missions.

Indeed, this would allow the implementation of more automated interaction pipelines, reducing the potential for mistakes and hopefully the iteration loop duration. Automatized traceability of computations, operations requests and acquired data would have been of great interest if CONSERT could have operated on multiple observations.

Currently, the CONSERT team is involved in the development of two new radars for the observation of Solar System small bodies. A Low Frequency Radar ${ }^{18,19,20}$ (LFR) is a direct heritage from CONSERT. Its new design will relax significantly the operational constraints due to clock synchronization between the lander and orbiter units. A High Frequency Radar 21, 19, 20 (HFR), inherited from ExoMars/WISDOM concept, is a mono-static step-frequency synthetic aperture radar specifically designed for small bodies' sub-surface tomography.

These radars developments have been initiated in the frame of the Asteroid Impact Mission ${ }^{21}$ (AIM) and are proposed on several future missions to support a better understanding of the asteroids and comets internal structure and compositions. Thanks to these current developments, we are now building analysis and traceability tools to manage our future payload operations.

\section{Acknowledgments}

Rosetta is an ESA mission with contributions from its member states and NASA.

Rosetta's Philae lander is provided by a consortium led by DLR, MPS, CNES and ASI.

The CONSERT instrument was designed, built and operated by IPAG, LATMOS and MPS and was financially supported by CNES, CNRS, UJF, DLR and MPS.

The authors would like to thank the teams of Rosetta (RMOC and RSGS) and Philae (LCC and SONC) for making possible the CONSERT operations.

They also thank the OSIRIS teams for the provision of the comet nucleus shape models.

Most of the computations presented in this paper were performed using the CIMENT infrastructure (https://ciment.ujf-grenoble.fr), including the Froggy platform, which is supported by the Rhône-Alpes region (GRANT CPER07_13 CIRA), the OSUG@2020 labex (reference ANR10 LABX56), the Equip@Meso project (reference ANR-10-EQPX-29-01) of the programme Investissements d'Avenir supervised by the Agence Nationale pour la Recherche and France-Grille (http://www.france-grilles.fr).

American Institute of Aeronautics and Astronautics 


\section{Références}

[1] S. Ulamec, et al., «Rosetta Lander - Landing and operations on comet 67P/Churyumov-Gerasimenko,» Acta Astronautica, vol. 125, pp. 80-91, 2016.

[2] H. Böhnhardt, et al., «The Philae lander mission and science overview,» Phil. Trans. R. Soc. A, vol. 375, 2017.

[3] A. Accomazzo, et al., «The final year of the Rosetta mission,» Acta Astronautica, vol. 136, pp. 354-359, 2017.

[4] W. Kofman, et al., «Properties of the 67P/Churyumov-Gerasimenko interior revealed by CONSERT radar,» Science, vol. 349, n⿳\%16247, 2015.

[5] A. Herique, et al., «Cosmochemical implications of CONSERT permittivity characterization of 67P/CG,» Monthly Notices of the Royal Astronomical Society, vol. 462, pp. 516-532, 2016.

[6] A. Herique, et al., «Philae localization from CONSERT/Rosetta measurement,» Planetary Space Science, vol. 117, pp. 475-484, 2015.

[7] P. Heinish, et al., «Attitude reconstruction of Rosetta's lander Philae using two-point magnetic field observations by ROMAP and RPC-MAG,» Acta Astronautica, vol. 125, pp. 174-182, 2016.

[8] W. Kofman, et al., «Comet nucleus sounding experiment by radio wave transmission,» Adv. Space Res., vol. 21, pp. 1589-1598, 1998.

[9] Y. Rogez, et al., «The CONSERT operations planning process for the Rosetta mission,» Acta Astronautica, vol. 125, pp. 212-233, 2016.

[10] L. O'Rourke, et al., "The search to identify and image the Philae Lander on the surface of comet 67P/Churyumov-Gerasimenko,» submitted to Acta Astronautica, 2018.

[11] R. Hahnel, et al., «CONSERT line-of-sight link budget simulator,» Planetary Space Science, vol. 111, pp. 5561, 2015.

[12] A. Moussi, et al., «The Philae Lander: Science planning and operations,» Acta Astronautica, vol. 125, pp. 92104, 2016.

[13] C. Vallat, et al., «The science planning process on the Rosetta mission,» Acta Astronautica, vol. 133, pp. 244257, 2017.

[14] M. Ashman, et al., «Rosetta science operations in support of the Philae mission,» Acta Astronautica, vol. 125, pp. 41-64, 2016.

[15] S. Ulamec, et al., «Rosetta Lander - Philae: landing preparations,» Acta Astronautica, vol. 107, pp. 79-86, 2015.

[16] A. Accomazzo, et al., «Rosetta mission operations for landing,» Acta Astronautica, vol. 125, pp. 30-40, 2016.

[17] P. V. d. Plas, et al., «MAPPS: a Science Planning tool supporting the ESA Solar System Missions,» chez SpaceOps 2016 Conference, 2016.

[18] C. Lange, et al., «MASCOT2 - A small body lander to investigate the interior of 65803 Didymos' moon in the Frame of AIDA/AIM,» chez International Astronautical Congress, 2017.

[19] A. Herique, et al., «Direct observations of asteroid interior and regolith structure: Science measurement requirements,» Advances in Space Research, p. In Press, 2017.

[20] A. Herique, et al., «Implications of implementing a radar package for asteroid subsurface investigations into the MASCOT nanoscale landing platform: from science requirements to baseline design,» Acta Astronautica (In Press), 2018.

[21] P. Michel, et al., «Science case for the Asteroid Impact Mission (AIM): A component of the Asteroid Impact \& Deflection Assessment (AIDA) mission,» Advances in Space Research, vol. 57, n \%112, pp. 2529-2547, 2016.

American Institute of Aeronautics and Astronautics 